\title{
Reaction Monitoring using Hyperpolarized NMR with Scaling of Heteronuclear Couplings by Optimal Tracking
}

\author{
Guannan Zhang ${ }^{a, \dagger}$, Franz Schilling ${ }^{b, \dagger}$, Steffen J. Glaser ${ }^{c *}$, Christian Hilty ${ }^{a *}$
}

${ }^{a}$ Chemistry Department, Texas A\&M University, 3255 TAMU, College Station, TX 77843, USA

${ }^{\mathrm{b}}$ Cancer Research UK Cambridge Institute, University of Cambridge, Li Ka Shing Centre, Cambridge CB2 0RE, United Kingdom

${ }^{\mathrm{c}}$ Department of Chemistry, Technische Universität München, 85747 Garching, Germany

${ }^{\dagger}$ Equal contribution

*Corresponding authors. E-mail: C. Hilty. chilty@ tamu.edu, S. J. Glaser. glaser@tum.de.

\begin{abstract}
Off-resonance decoupling using the method of Scaling of Heteronuclear Couplings by Optimal Tracking (SHOT) enables determination of heteronuclear correlations of chemical shifts in single scan NMR spectra. Through modulation of $J$-coupling evolution by shaped radio frequency pulses, off resonance decoupling using SHOT pulses causes a user-defined dependence of the observed $J$-splitting, such as the splitting of ${ }^{13} \mathrm{C}$ peaks, on the chemical shift offset of coupled nuclei, such as ${ }^{1} \mathrm{H}$. Because a decoupling experiment requires only a single scan, this method is suitable for characterizing on-going chemical reactions using hyperpolarization by dissolution dynamic nuclear polarization (D-DNP). We demonstrate the calculation of $\left[{ }^{13} \mathrm{C},{ }^{1} \mathrm{H}\right]$ chemical shift correlations of the carbanionic active sites from hyperpolarized styrene polymerized using sodium naphthalene as an initiator. While off resonance decoupling by SHOT pulses does not enhance the resolution in the same way as a 2D NMR spectrum would, the ability to obtain the correlations in single scans makes this method ideal for determination 1
\end{abstract}

(C) 2016. This manuscript version is made available under the Elsevier user license http://www.elsevier.com/open-access/userlicense/1.0/ 
of chemical shifts in on-going reactions on the second time scale. In addition, we present a novel SHOT pulse that allows to scale $J$-splittings $50 \%$ larger than the respective $J$-coupling constant. This feature can be used to enhance the resolution of the indirectly detected chemical shift and reduce peak overlap, as demonstrated in a model reaction between p-anisaldehyde and isobutylamine. For both pulses, the accuracy is evaluated under changing signal-to-noise ratios (SNR) of the peaks from reactants and reaction products, with an overall standard deviation of chemical shift differences compared to reference spectra of $0.02 \mathrm{ppm}$ when measured on a $400 \mathrm{MHz}$ NMR spectrometer. Notably, the appearance of decoupling side-bands, which scale with peak intensity, appears to be of secondary importance.

\section{Keywords}

Dissolution dynamic nuclear polarization, nuclear magnetic resonance, correlation spectroscopy, optimal control methods 


\section{Introduction}

Hyperpolarized nuclear spin states now can be quite readily created for liquid state samples using technology such as dissolution dynamic nuclear polarization (D-DNP) [1]. The resulting signal gain of several orders of magnitude in a single scan or a series of scans in rapid succession, provides new impetus for the application of NMR to the study of non-equilibrium processes using stopped-flow techniques [2-4]. With its high sensitivity to structural change, NMR spectroscopy in particular assisted by hyperpolarization offers the promise of enabling the characterization of short-lived species in chemical and biochemical reactions $[5,6]$. At the same time, the rapid time evolution in stopped-flow experiments precludes the application of the traditional multi-dimensional NMR techniques that allow for the determination of chemical shift correlations, which often are crucial structural indicators [7]. As such, stopped-flow NMR a priori lacks access to one of the most powerful types of information available from NMR spectroscopy. Various methods however allow fully or partially recovering this information. Complete multi-dimensional data sets can be acquired in a single scan by spatial encoding of indirect dimensions, by the use of pulsed field gradient based techniques [8-10]. In a small number of scans, combinatorial selective excitation such as in Hadamard spectroscopy likewise can be used to explore a second spectral dimension [11,12]. Chemical shift correlations are further available from offresonance decoupling, which imparts a change in observed $J$-splitting dependent on the chemical shift of an indirectly observed nucleus $[13,14]$.

In previous work, some of the authors of this manuscript have developed an efficient optimization technique for generating hetero-nuclear decoupling sequences [15-17] based on principles of optimal control theory $[18,19]$. This highly flexible approach makes it also possible to design decoupling sequences that produce a pre-defined chemical shift dependence of the observed multiplet splitting. Pulses developed by this method, termed Scaling of Heteronuclear Couplings by Optimal Tracking (SHOT), have been demonstrated to allow reliable determination of chemical shifts combined with 
rapid implementation once pulses have been calculated [16,20]. When applied to stopped-flow NMR techniques, a major advantage of SHOT based determination of indirect chemical shift is the need for only a single scan to determine the chemical shift from all offset frequencies. Two pulses that exhibit a piecewise linear dependence of the scaled $J$-splitting on chemical shift have subsequently been used in combination with dissolution dynamic nuclear polarization.

Here, we demonstrate the application of SHOT pulses to obtain chemical shift correlations from series of spectra typically used for the determination of reaction kinetics and mechanisms from D-DNP hyperpolarized samples. Specifically, we demonstrate the calculation of ${ }^{1} \mathrm{H}$ chemical shifts from ${ }^{13} \mathrm{C}$ spectra acquired of a transient carbanionic species in a catalyzed polymerization reaction of styrene. In addition, we extend the capability of SHOT pulses to allow for the scaling of $J$-splittings larger than the actual $J$-coupling constant. Subsequently, we evaluate the accuracy of the indirect chemical shift determination as a function of changing signal-to-noise ratio in these data sets.

\section{Materials and methods}

\subsection{Dynamic Nuclear Polarization}

For DNP experiments of the catalyzed polymerization of styrene or the reaction of p-anisaldehyde, a $40 \mu \mathrm{L}$ or $25 \mu \mathrm{L}$ sample of the reactant styrene or p-anisaldehyde, which both are self-glassing, was mixed with $15 \mathrm{mM}$ of the free radical $\alpha, \gamma$-Bisdiphenylene- $\beta$-phenylallyl (BDPA; Sigma-Aldrich, St. Louis, MO). The sample was hyperpolarized for 3 hours at $1.4 \mathrm{~K}$ in a HyperSense system (Oxford Instruments, Abingdon, UK), by irradiating microwaves $(60 \mathrm{~mW})$ at a frequency of $93.965 \mathrm{GHz}$ in a 3.35 T magnetic field. Subsequently, the hyperpolarized sample was quickly dissolved in pre-heated dioxane or trifluoroethanol (Alfa Aesar, Ward Hill, MA), and injected rapidly into a $5 \mathrm{~mm}$ NMR tube installed in an NMR instrument (Bruker Biospin, Billerica, MA) by a gas driven injection device [21] at a temperature of $298 \mathrm{~K}-300 \mathrm{~K}$. Because the polymerization reaction is sensitive to oxygen and water, 
the transfer line in the DNP polarizer, the injection system and the NMR tube were purged with nitrogen gas before preloaded the catalyst solution. In the NMR tube, the hyperpolarized sample was mixed with $35 \mu \mathrm{L}$ catalyst solution or $25 \mu \mathrm{L}$ isobutylamine (Alfa Aesar, Ward Hill, MA). The catalyst solution is sodium naphthalenide, which was prepared by dissolving $0.5 \mathrm{~g}$ sodium film and $2 \mathrm{~g}$ naphthalene in $10 \mathrm{~mL}$ tetrahydrofuran (THF) solution and sonicated for $5 \mathrm{~min}$ at a temperature of 298 K.

\subsection{SHOT pulses}

SHOT pulses are optimized such that observed $J$-splittings under off resonance decoupling by SHOT pulses $\left(J_{\text {SHOT }}\right)$ depend linearly on frequency offset $(v)$ [20]. Equation 1 describes this relationship, with the $J$-splitting varying between zero and $s_{\mathrm{J}} \times J_{\text {opt }}$ for offsets between $-v_{\max }$ and $v_{\max }$,

$$
J_{S H O T}(\nu)=s_{J} \times J_{o p t} \frac{\nu+\nu_{\max }}{2 \nu_{\max }}
$$

Here, $s_{\mathrm{J}}$ is an arbitrary scaling factor that can also be larger than 1 . For a nominal coupling $J_{\mathrm{opt}}=160$ $\mathrm{Hz}$, a first pulse with $s_{\mathrm{J}}=1$ and $v_{\max }=1250 \mathrm{~Hz}$ was used as described previously, resulting in a maximal $J$-splitting of $160 \mathrm{~Hz}$ for $v=v_{\max }$. A second pulse with a higher bandwidth $v_{\max }=2000 \mathrm{~Hz}$ and a scaling factor $s_{\mathrm{J}}=1.5$ was newly computed by the method described. For the case of $s_{\mathrm{J}}=1.5$, a maximum $J$-splitting of $240 \mathrm{~Hz}$ occurs at $v=v_{\max }$ in the optimization algorithm, which is $50 \%$ larger than the actual coupling constants of about $J_{\mathrm{opt}}=160 \mathrm{~Hz}$ for which the SHOT was optimized. The maximum required radio frequency (rf) amplitudes $(\gamma / 2 \pi) B_{1, \text { max }}$ for the two pulses were $1.64 \mathrm{kHz}$ and $2.1 \mathrm{kHz}$, respectively. The value for $J_{\mathrm{opt}}$ was chosen to lie within the typical range of direct heteronuclear carbon-proton couplings, and it has been shown that the SHOT off-resonance decoupling profile is very robust against deviations between the actual experimental coupling constants $J_{\text {opt }}$ [20]. A guide to the implementation of SHOT pulses presented in this paper on a typical NMR spectrometer is given in the Supporting Information. 


\subsection{NMR spectroscopy}

The pulse sequence for ${ }^{13} \mathrm{C}$ detected hyperpolarized NMR experiments was (trigger $-\left[G_{\mathrm{z}}-\alpha-\right.$ acquire $_{\times n}$ ). For the catalyzed polymerization of styrene, a total of $n=8$ transients were acquired within $3 \mathrm{~s}$. For the experiment with p-anisaldehyde, a total of $n=15$ transients were acquired over a duration of 12 s. $G_{\mathrm{Z}}$ was a pulsed field gradient $(97 \mathrm{G} / \mathrm{cm}, 1 \mathrm{~ms})$ applied for attenuation of coherences from previous scans. $\alpha$ was an excitation pulse of strength $\gamma B_{1}=28.57 \mathrm{kHz}$ corresponding to a flip angle of 20 degrees for the styrene experiment or 13 degrees for the p-anisaldehyde experiment. In each scan, a total of 12,800 complex points (10 times the number of digitization points of the SHOT pulses) was acquired. During acquisition, ${ }^{1} \mathrm{H}$ off resonance decoupling by one of the two SHOT pulses was applied $\left(s_{\mathrm{J}}=1\right.$ for the reaction with styrene and $s_{\mathrm{J}}=1$ or 1.5 for the reaction with p-anisaldehyde, see below). The acquisition time during each transient is $256 \mathrm{~ms}$, which is the same as the SHOT pulse length. The SHOT pulse is applied once for each transient acquisition.

The proton and carbon chemical shifts of both the reactant and product were calibrated to the ${ }^{1} \mathrm{H}$ signal and ${ }^{13} \mathrm{C}$ signal of tetramethylsilane (TMS) at $0 \mathrm{ppm}$. On the ${ }^{1} \mathrm{H}$ channel, the transmitter frequency offset to the frequency of $0 \mathrm{ppm}$ was $2286.02 \mathrm{~Hz}$ for the catalyzed polymerization reaction. The ${ }^{1} \mathrm{H}$ chemical shifts of the transient carbanionic site were verified from the ${ }^{1} \mathrm{H}$ spectrum acquired immediately after a DNP experiment, while the reaction was still progressing. The ${ }^{1} \mathrm{H}$ chemical shifts were referenced to the solvent resonance of dioxane. The chemical shift of dioxane was calibrated to the ${ }^{1} \mathrm{H}$ signal of TMS at $0 \mathrm{ppm}$ using a separate sample. For the experiments with p-anisaldehyde, the transmitter frequency offset to the frequency of $0 \mathrm{ppm}$ was $2729.23 \mathrm{~Hz}$ for the experiment with the pulse using $s_{\mathrm{J}}=1$ and $1908.63 \mathrm{~Hz}$ for the experiment with the pulse using $s_{\mathrm{J}}=1.5$ on the ${ }^{1} \mathrm{H}$ channel. To verify the actual proton chemical shifts, a proton spectrum was acquired immediately after a DNP experiment under the same experimental conditions. Because isobutylamine was in excess, the reactant was fully consumed. Therefore, the actual proton chemical shifts of the reactant peaks were verified 
using another sample, in which the concentration of p-anisaldehyde was the same as the concentration used in the DNP experiment.

Data was Fourier transformed and zero-filled to 65536 points or 131072 points and an exponential window function was applied (line broadening of $10 \mathrm{~Hz}$ or $0 \mathrm{~Hz}$ ) for the experiments with styrene and p-anisaldehyde, respectively. Peak positions for each of the doublet peaks were picked using the Topspin 3 software (Bruker Biospin). Indirect ${ }^{1} \mathrm{H}$ chemical shifts were back-calculated based on Eq. (1).

\section{Results and discussion}

Since the chemical shift of a nucleus decoupled by a SHOT pulse can be calculated from a single 1D spectrum, this technique would open the possibility for determining chemical shift correlations in non-equilibrium samples. The time resolution in this case is given by the acquisition time of a single 1D spectrum, which is on the order of a second or less. To evaluate the performance of the SHOT pulse for this purpose, the anionic polymerization of hyperpolarized styrene was studied as a model reaction using $\left\{{ }^{1} \mathrm{H}\right\}{ }^{13} \mathrm{C}$ NMR $[22,23]$. In this reaction, the coupling constants of the peaks of both the reactant and the generated species with carbanionic active site range from $160 \mathrm{~Hz}$ to $154 \mathrm{~Hz}$, which is within the optimized $J$-splitting range of the SHOT pulse. Series of $1 \mathrm{D}^{13} \mathrm{C}$ spectra during this reaction were acquired with the ${ }^{1} \mathrm{H}$ SHOT pulse, as shown in Fig. 1a. In these spectra, ${ }^{13} \mathrm{C}$ signals from the transient carbanionic site, where monomer addition to the nascent polymer chain occurs, can be identified as 1', $2^{\prime}, 3^{\prime} \mathrm{b}, 4^{\prime}, 5^{\prime} \mathrm{a}$ and $5^{\prime} \mathrm{b}$. Based on the observed $J$-splittings of these ${ }^{13} \mathrm{C}$ peaks, the correlated ${ }^{1} \mathrm{H}$ chemical shifts were calculated using Eq. (1) [20]. For example, the peaks of C5'a and C5'b of the first scan in Fig. 1a stem from the phenyl ring of the transient carbanionic species. These peaks show observed $J$ splittings $J_{\mathrm{SHOT}}=67 \mathrm{~Hz}$ and $J_{\mathrm{SHOT}}=75 \mathrm{~Hz}$. The resulting ${ }^{1} \mathrm{H}$ chemical shifts are calculated as $5.21 \mathrm{ppm}$ and $5.51 \mathrm{ppm}$, respectively. The correlated ${ }^{1} \mathrm{H}$ chemical shifts for all of the ${ }^{13} \mathrm{C}$ atoms are shown in 
Supporting Information Table S1.

Since the SHOT pulse is applied for each scan, the ${ }^{1} \mathrm{H}$ chemical shifts of the carbanionic species in the indirect dimension can be calculated for each time point. In this reaction, large scan-to-scan chemical shift changes are not expected. Accordingly, for instance the ${ }^{1} \mathrm{H}$ chemical shifts of the peak of C4' in the first four scans at time points $0,0.4,0.8$ and $1.6 \mathrm{~s}$, are calculated as 4.68, 4.66, 4.63 and 4.63 ppm, corresponding to a variation of less than $20 \mathrm{~Hz}$.

a)

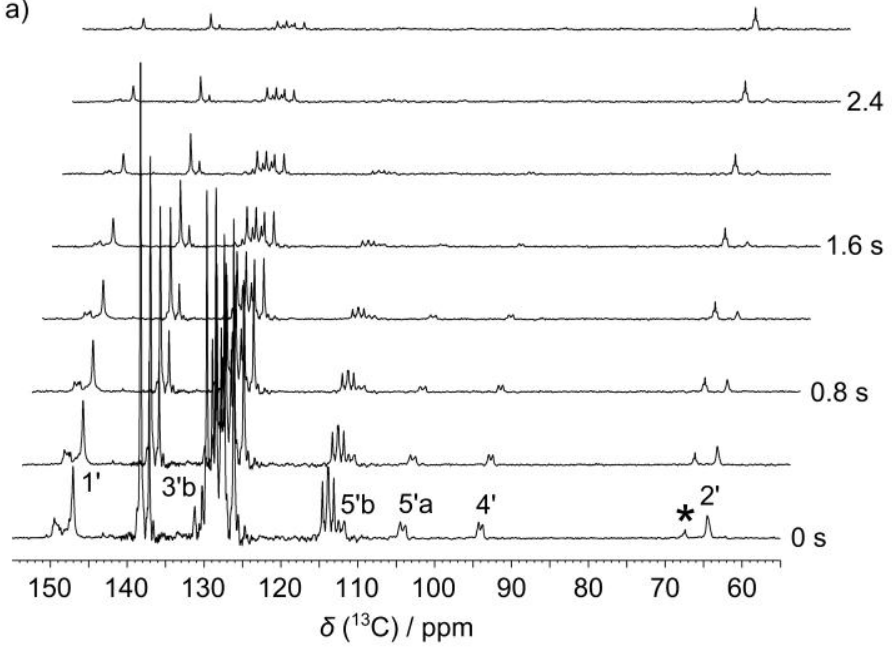

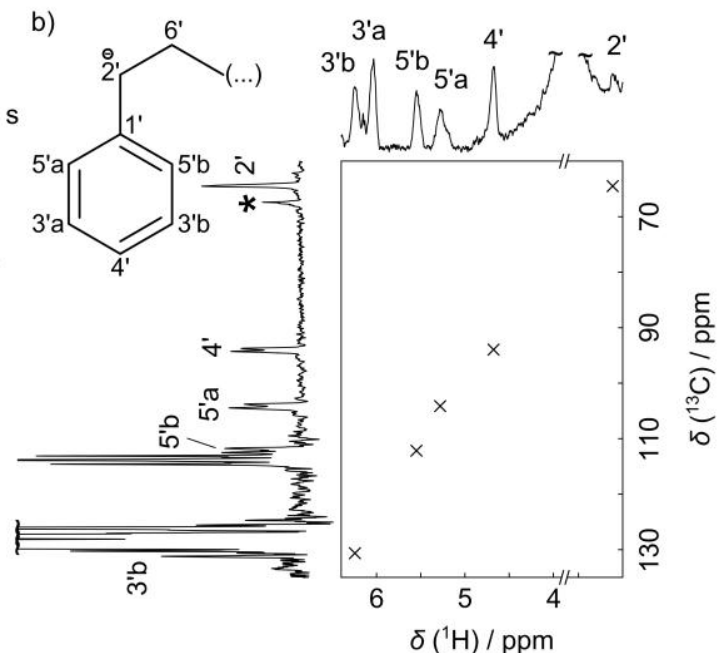

Fig. 1. a) Stacked plots of a series of ${ }^{13} \mathrm{C}$ spectra from hyperpolarized styrene mixed with polymerization initiator $\mathrm{NaC}_{10} \mathrm{H}_{8}$, acquired with the ${ }^{1} \mathrm{H}$ SHOT pulse of $J_{\mathrm{opt}}=160 \mathrm{~Hz}$ and $s_{J}=1$. The dioxane solvent peak is designated with an asterisk. The first 8 scans of the spectra at time intervals of $400 \mathrm{~ms}$ are shown. b) 2D representation of chemical shifts calculated from the first scan of (a), that correlates a $1 \mathrm{D}$ hyperpolarized ${ }^{13} \mathrm{C}$ spectrum and thermal ${ }^{1} \mathrm{H}$ spectrum measured separately. The labeled ${ }^{13} \mathrm{C}$ and ${ }^{1} \mathrm{H}$ resonances correspond to the carbanionic active polymerization site.

To illustrate the use of these correlations for chemical shift assignment, a 2D representation of the data obtained from this experiment is plotted in Fig. 1b. Here, a hyperpolarized ${ }^{13} \mathrm{C}$ and a thermal ${ }^{1} \mathrm{H}$ 1D spectrum are correlated by placing cross marks at the chemical shifts calculated from the SHOT experiment. This representation is intended to resemble a familiar 2D NMR spectrum, even if acquired 
from hyperpolarized ${ }^{13} \mathrm{C}$ spins. Since this correlation is calculated from the 1D SHOT spectrum, it does not increase the resolution as a conventional 2D NMR data set would. This limitation can be seen in peak 3 'a, which is shown in the proton spectrum but is not observed in the hyperpolarized ${ }^{13} \mathrm{C}$ spectrum because it is overlapped with the monomer peaks [24]. However, a SHOT based correlation spectrum is available in each scan in a time resolved data set.

Previous DNP experiments that enabled measurement of the kinetics of this styrene polymerization reaction did not allow the determination of ${ }^{1} \mathrm{H}$ chemical shifts from the same data set [6]. Rather, the origin of transient peaks were identified based on known chemical shifts, as well as the correlation of signals between the reactant and the reaction product through selective inversion experiments. However, with the SHOT pulse, as shown here, the correlation could be obtained within seconds. These chemical shift correlations greatly facilitate the assignment of peaks in such transient DNP-NMR spectra, while retaining the ability for kinetic measurements based on peak intensities and for detecting species arising transiently during a reaction.
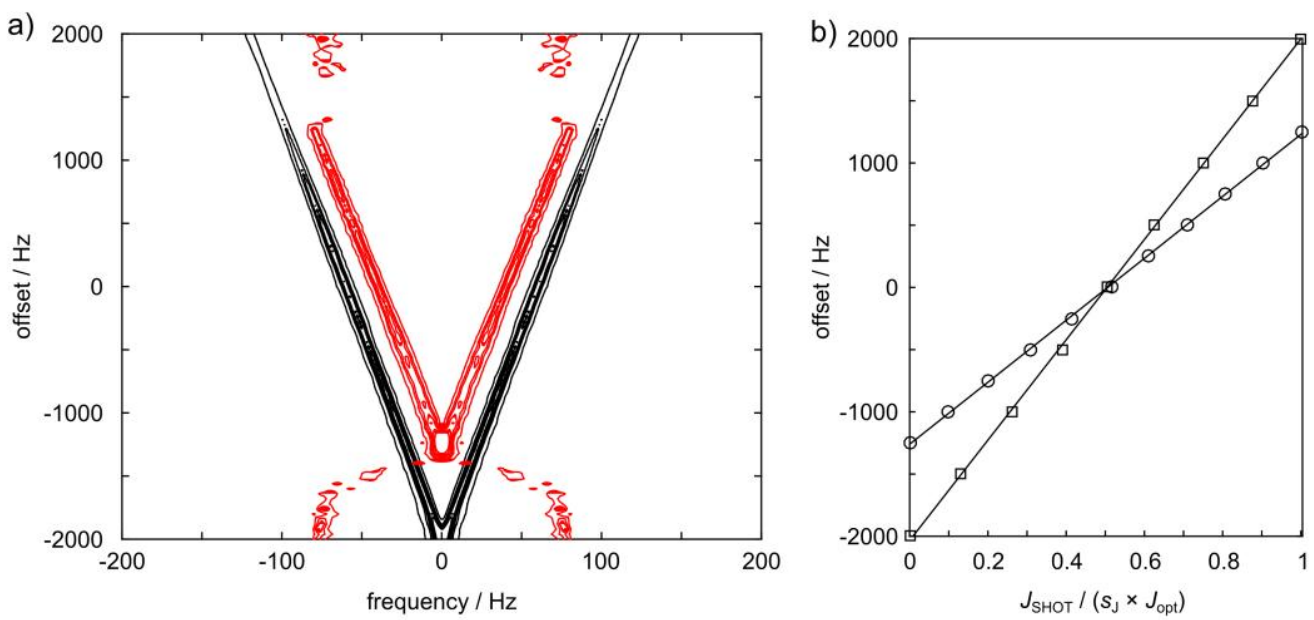

Fig. 2. a) Contour plot of the simulated frequency profiles of the SHOT pulses with $J_{\text {opt }}=160 \mathrm{~Hz}$ and $s_{J}=1$ (red) or $s_{\jmath}=1.5$ (black) as a function of the transmitter offset frequency. The frequency profiles are simulated for actual coupling constants ranging from $174 \mathrm{~Hz}$ to $145 \mathrm{~Hz}$ (in steps of $1 \mathrm{~Hz}$ ). Contour levels are at [0.2, 0.3, 0.35, $0.4,0.45,0.5]$ of the maximum decoupled peak amplitude. When the spins are not in the optimized bandwidth, the pulse with $s J=1$ shows a non-defined off-resonance decoupling pattern. b) The theoretical $J$-splitting values 
determined from Eq. (1) (solid lines) and the observed $J$-splitting values as a function of the ${ }^{1} \mathrm{H}$ off resonance decoupling offset using SHOT pulses with $s_{J}=1$ (circles) and $s_{J}=1.5$ (squares) for the aldehyde group $\left(J_{\text {actual }}=\right.$ $174 \mathrm{~Hz}$ ) of p-anisaldehyde, measured on a $400 \mathrm{MHz}$ NMR spectrometer.

The power of off-resonance decoupling by SHOT pulses for chemical shift determination derives from the ability to define an arbitrary dependence between the frequency offset of the decoupled nucleus and the $J$-splitting of the detected nucleus. With the $J$-splittings obtained from a pulse that yields a maximum splitting of $160 \mathrm{~Hz}$ (Fig. 2) [20], the peaks of the carbanionic species are resolved in the spectra styrene polymerization. However, in the general application it is expected that peak shifts due to the decoupling result in signal overlap for a certain proportion of spectra. Signal overlap in such a case could however readily be avoided by scaling to different $J$-splittings. Here, we extend the repertoire of SHOT pulses with a design that can scale a $J$-splitting to a value that is larger than the original $J$-coupling constant. In this case, we chose $s_{\mathrm{J}} \times J_{\mathrm{opt}}=240 \mathrm{~Hz}$, which is about $50 \%$ larger than typical ${ }^{13} \mathrm{C}-{ }^{1} \mathrm{H}$ coupling constants. The larger slope of $\Delta J_{\text {SHOT }} / \Delta v$ of the new pulse (Fig. 2b) increases the sensitivity of the chemical shift determined in the indirect dimension. The relationship between the frequency offset and $J_{\mathrm{SHOT}}$ of this pulse was chosen to be a linear function (Eq. (1)), as was the case for the previously used pulse. This linear relationship is intended to provide a uniform slope and hence a uniform precision of the chemical shift determination in the indirect dimension and in addition facilitate data analysis. The simulated frequency profiles of the two pulses are compared in Fig. 2a. The two branches in the figure represent the contour lines from the peaks of the doublet of two heteronuclear coupled spins, when the decoupling pulse is applied at the frequency offset indicated on the vertical axis. Because of the robustness of SHOT pulses to $J$ variation [20], the simulated frequency profiles for actual coupling constants ranging from $174 \mathrm{~Hz}$ to $145 \mathrm{~Hz}$ show little variation in the decoupling pattern of SHOT pulses (Fig. 2a). Application of off-resonance decoupling to molecules with a different range of actual coupling constant is possible but would require re-optimisation of the 
pulses to the actual range of coupling constants.

For comparison, the experimental performance of the SHOT pulses was measured using a concentrated sample of p-anisaldehyde, without DNP hyperpolarization (Fig. 2b). The observed $J$ splittings $\left(J_{\mathrm{SHOT}}\right)$ for the aldehyde signal, which has an actual $J$-splitting of $174 \mathrm{~Hz}$, are plotted as a function of transmitter frequency offset in the indirect ${ }^{1} \mathrm{H}$ dimension. The linear dependence of the observed $J$-splitting on the transmitter offset can be seen as designed. In the case of the SHOT pulse with $s_{\mathrm{J}} \times J_{\mathrm{opt}}=160 \mathrm{~Hz}$, all of the observed $J$-splittings represent an apparent down-scaling of the actual coupling constant, as would normally be expected in an experiment employing off-resonance decoupling [25]. However, for the pulse with $s_{\mathrm{J}} \times J_{\mathrm{opt}}=240 \mathrm{~Hz}$, the two points with highest offset represent an apparent up-scaling of the actual coupling constant.

a)

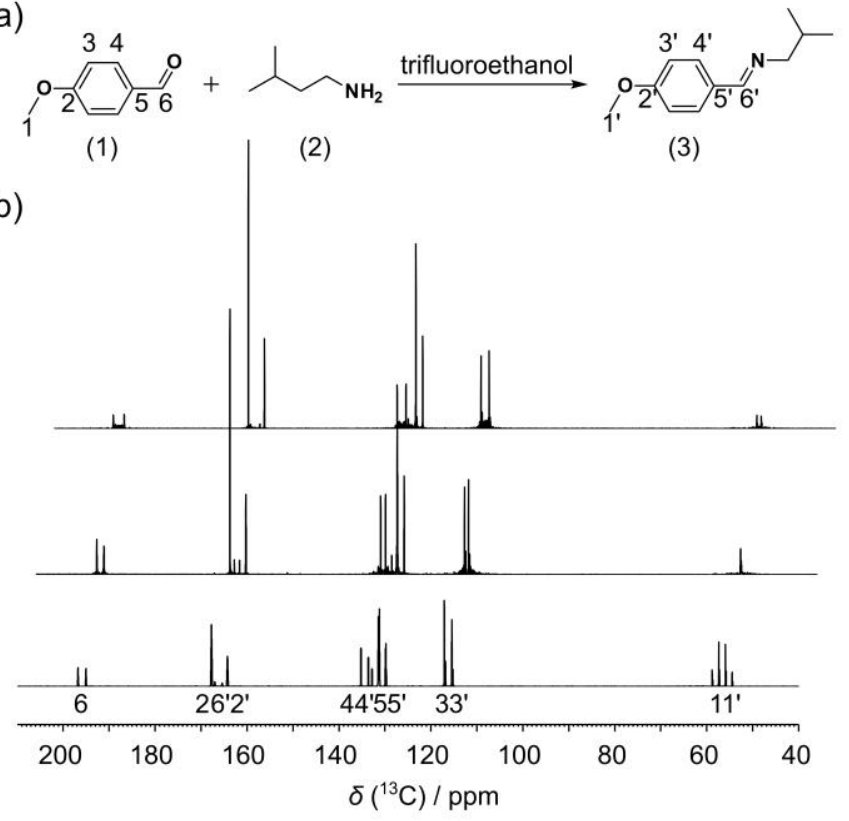

c)
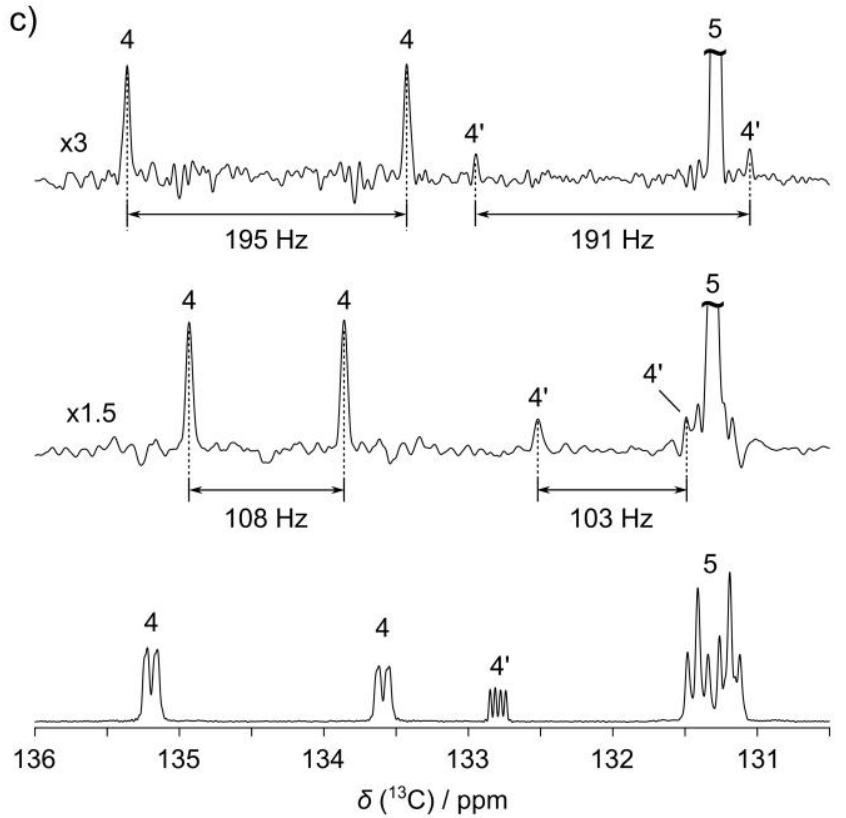

Fig. 3. a) The chemical reaction between $p$-anisaldehyde (1) and isobutylamine (2) using trifluoroethanol as solvent. b) Hyperpolarized ${ }^{13} \mathrm{C}$ NMR reference spectrum without decoupling (bottom), with ${ }^{1} \mathrm{H}$ SHOT pulses of $J_{\mathrm{opt}}=160 \mathrm{~Hz}$ and $s_{\jmath}=1$ (middle) or $s_{\jmath}=1.5$ (top). c) Expanded views of (b) are shown for peak 4, 4' and 5.

The difference between the two pulses is seen in the reaction between p-anisaldehyde and 
isobutylamine, where a Schiff base N-(4-methoxybenzylidene)-2-methylpropan-1-amine is formed (Fig. 3a). The spectra from the third scan with two different SHOT pulses, as well as one spectrum without decoupling, are shown in Fig. 3b, and are expanded around peak 4 in Fig. 3c (see also Fig. S1 and S2 in Supporting Information). Here, one of the C4' peaks overlaps with C5 both in the spectrum without decoupling, as well as in the decoupled spectrum using the pulse with $s_{\mathrm{J}} \times J_{\mathrm{opt}}=160 \mathrm{~Hz}$. This overlap is resolved by the larger observed $J$-splitting of $191 \mathrm{~Hz}$ when using the pulse with $S_{\mathrm{J}} \times J_{\text {opt }}=$ $240 \mathrm{~Hz}$. According to Eq. (1), the ratio of the observed $J$-splittings of the same ${ }^{13} \mathrm{C}$ peak in the decoupled spectra (top and middle) depends on their individual $s \mathrm{~J}, v_{\max }$ and frequency offset $(v)$ which is the difference between the calculated ${ }^{1} \mathrm{H}$ frequency and transmitter frequency. For instance, the frequency offsets of C4 are $v=1256 \mathrm{~Hz}$ (top) and $436 \mathrm{~Hz}$ (middle). With $v_{\max }=2000 \mathrm{~Hz}$ (top) or 1250 $\mathrm{Hz}$ (middle) and $s_{\mathrm{J}}=1.5$ (top) or 1 (middle), the calculated $J_{\mathrm{SHOT}}$ ratio of $\mathrm{C} 4$ in the top and middle spectrum is 1.8 as observed in Fig. 3c.
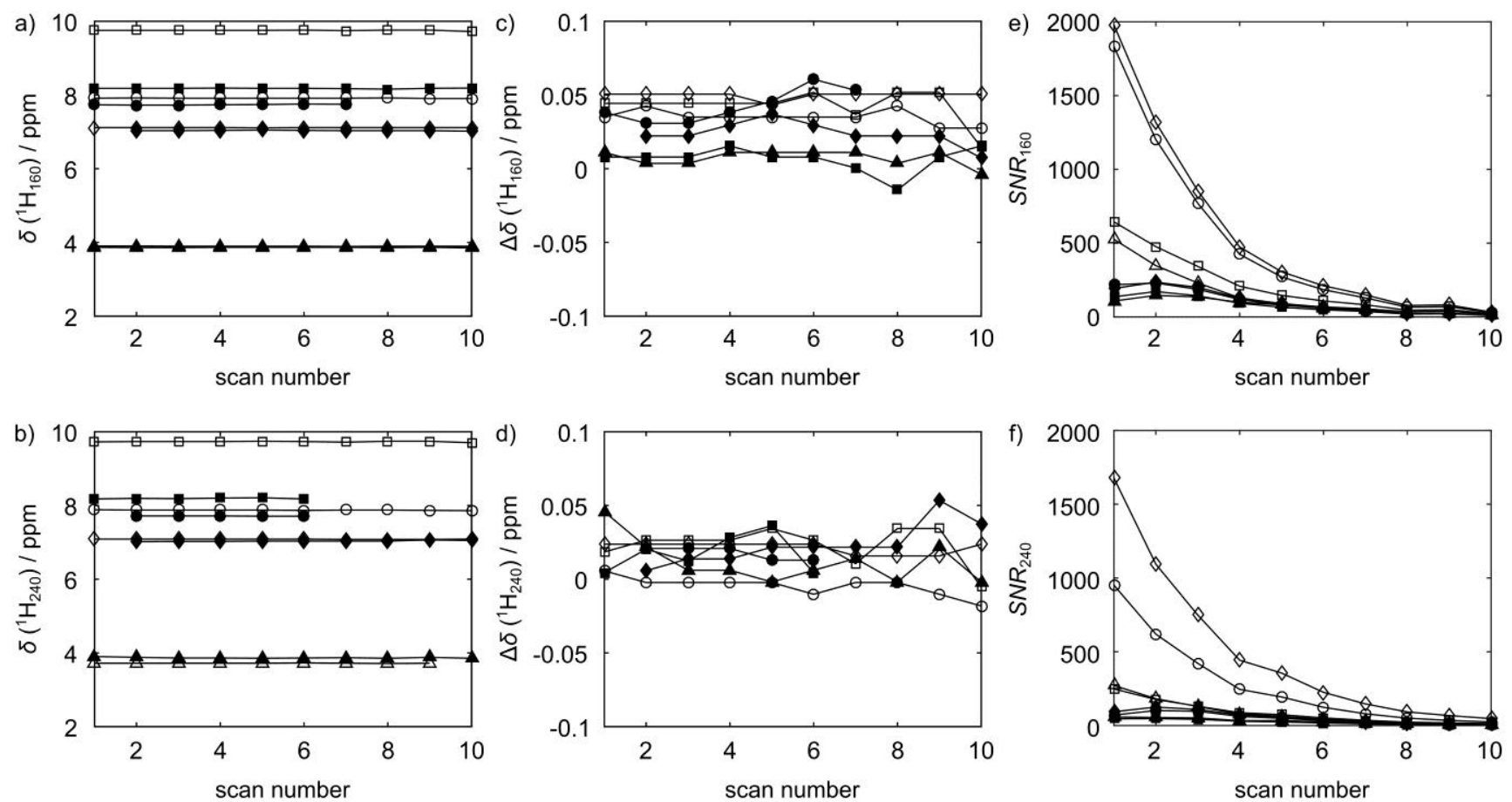

Fig. 4. a) The calculated ${ }^{1} \mathrm{H}$ chemical shift $\delta\left({ }^{1} \mathrm{H}\right)$ from the ${ }^{13} \mathrm{C}$ experiment with the ${ }^{1} \mathrm{H}$ SHOT pulse of $J_{\mathrm{opt}}=160$ $\mathrm{Hz}$ and $s_{\lrcorner}=1$, shown as a function of scan number. b) The same as (a), but using the SHOT pulse with $s_{J}=1.5$. 
c) ${ }^{1} \mathrm{H}$ chemical shift difference $\Delta \delta\left({ }^{1} \mathrm{H}\right)$ between the calculated value of (a) and the real value from a thermal measurement, shown as a function of scan number. d) $\Delta \delta\left({ }^{1} \mathrm{H}\right)$ between the calculated value of (b) and the real value from a thermal measurement, shown as a function of scan number. e) The SNR of each corresponding ${ }^{13} \mathrm{C}$ resonance using the SHOT pulse with $s \jmath=1$, shown as a function of scan number. f) The same as (e), but using the SHOT pulse with $s_{J}=1.5$. Carbons $1,1^{\prime}, 3,3$, 4, 4', 6, 6' are indicated by open and filled triangle, diamond, circle and square. In (a)-(d), no data points are displayed when no signal was detectable.

The increased $J$-splitting appears at the expense of also increased spurious signals ("sidebands"), which add to the apparent noise in the spectrum. In Fig. 3c, the apparent signal to noise ratio (SNR) for C4 (inset) near the peak with the pulse of $s_{\mathrm{J}} \times J_{\mathrm{opt}}=240 \mathrm{~Hz}$ and with the pulse of $s_{\mathrm{J}} \times J_{\mathrm{opt}}=160 \mathrm{~Hz}$ are calculated as 20 and 25, respectively. Because of the side band signals, the SNR away from the peak which are $S N R_{240}=421$ and $S N R_{160}=771$ is larger than the apparent SNR near the peak for C4. These side band signals however scale with the main peak intensity, without degrading the limit of detection for the peak observed. For this reason, the apparent SNR of the C4 peak is stable for both pulses from scan 1 to scan 7, with values of $19.5 \pm 0.5$ for the pulse with $s \mathrm{~J} \times J_{\mathrm{opt}}=240 \mathrm{~Hz}$ and $24.5 \pm 0.5$ for the pulse with $s_{\mathrm{J}} \times J_{\mathrm{opt}}=160 \mathrm{~Hz}$, but decreases in the later scans. Side bands could, however, interfere with nearby signals that are small.

From the $J$-splittings measured in each $1 \mathrm{D}^{13} \mathrm{C}$ spectrum (Fig. 3b), proton chemical shifts of both the reactant and the reaction product were determined using Eq. (1) (Supporting Information Tables 2 and 3). Since the ${ }^{13} \mathrm{C}$ data sets contain signals from reactant and reaction product in the first 10 scans, the corresponding ${ }^{1} \mathrm{H}$ chemical shifts for these species can be calculated from each scan and should remain constant throughout the reaction as shown in Fig. 4a and b. The precision and accuracy of the correlated ${ }^{1} \mathrm{H}$ chemical shifts can be evaluated by comparing the chemical shift values among the different scans, as well as by comparing with ${ }^{1} \mathrm{H}$ NMR spectra of the respective compounds. Fig. 4c and $\mathrm{d}$ show the difference between the chemical shifts determined from the experiments with SHOT 
pulses and the ${ }^{1} \mathrm{H}$ NMR reference experiment, apart from the methyl group (1), for which the ${ }^{1} \mathrm{H}$ chemical shift overlaps with the solvent resonance in the reference spectrum. The overall standard deviation of these difference values is $0.02 \mathrm{ppm}$, representing a measure for the accuracy of the determination of the indirect ${ }^{1} \mathrm{H}$ chemical shift. Even though the ratio of signal to stochastic noise decreases as scan number increases (Fig. 4e and $\mathrm{f}$ ), the correlated ${ }^{1} \mathrm{H}$ chemical shift in the indirect dimension can still be calculated accurately for each scan.

\section{Conclusions}

Chemical shift correlations are obtained from a single NMR spectrum, which is useful in combination with hyperpolarization by D-DNP for monitoring non-equilibrium chemical reactions, with the goal of determining the identity of transient species and reaction mechanisms. Under offresonance decoupling by SHOT pulses, the calculation of indirectly detected chemical shift is straight forward due to the linear relationship between the observed $J$-splitting and frequency offset and due to the uniform slope the precision of the chemical shift determination is uniform in the entire frequency range for which the pulses were developed. Moreover, the chemical shifts can be estimated even without precise knowledge of actual coupling constants. In addition, SHOT pulses can scale the observed $J$-splittings in an NMR spectrum to values larger than the actual coupling constant which was demonstrated here for the first time. This feature is useful to resolve overlap in some cases. In view of the robustness and, once pulses have been computed, simple implementation of this method, the scope of its application can be extended to many small and medium sized molecules. It may be useful in areas such as analyzing enzymatic and chemical reaction intermediates, as well as studying the fate of in vivo metabolites over time. 


\section{Acknowledgements}

C. H. gratefully acknowledges financial support from the Welch Foundation (Grant A-1658), the National Science Foundation (Grant CHE-1362691) and the Ji and Li Family Foundation. S. J. G. acknowledges support from the Deutsche Forschungsgemeinschaft (DFG; Grant GI 203/7-2). F. S. is in receipt of funding from the Alexander von Humboldt Foundation in the form of a Feodor Lynen Research Fellowship.

\section{Appendix A. Supplementary material}

Supplementary data associated with this article can be found, in the online version.

Additional information as noted in text. The SHOT pulses are available for download at http://www.ocnmr.ch.tum.de/en/downloads.

\section{References}

[1] J.H. Ardenkjaer-Larsen, B. Fridlund, A. Gram, G. Hansson, L. Hansson, M.H. Lerche, R. Servin, M. Thaning, K. Golman, Increase in signal-to-noise ratio of $>10,000$ times in liquid-state NMR, Proc. Natl. Acad. Sci. 100 (2003) 10158-10163. doi:10.1073/pnas.1733835100.

[2] S. Bowen, C. Hilty, Time Resolved Dynamic Nuclear Polarization Enhanced NMR Spectroscopy, Angew. Chem. Int. Ed. 47 (2008) 5235-5237. doi:10.1002/anie.200801492.

[3] H. Zeng, Y. Lee, C. Hilty, Quantitative Rate Determination by Dynamic Nuclear Polarization Enhanced NMR of a Diels-Alder Reaction, Anal. Chem. 82 (2010) 8897-8902. doi:10.1021/ac101670n.

[4] C.-H. Chen, W.-C. Shih, C. Hilty, In Situ Determination of Tacticity, Deactivation, and Kinetics in $\left[\right.$ rac- $\left.\left(\mathrm{C}_{2} \mathrm{H}_{4}(1 \text {-Indenyl })_{2}\right) \mathrm{ZrMe}\right]\left[\mathrm{B}\left(\mathrm{C}_{6} \mathrm{~F}_{5}\right)_{4}\right]$ and $\left[\mathrm{Cp}_{2} \mathrm{ZrMe}\right]\left[\mathrm{B}\left(\mathrm{C}_{6} \mathrm{~F}_{5}\right)_{4}\right]$-Catalyzed Polymerization of 1-Hexene Using ${ }^{13}$ C Hyperpolarized NMR, J. Am. Chem. Soc. 137 (2015) 6965-6971. doi:10.1021/jacs.5b04479.

[5] P.R. Jensen, S. Meier, J.H. Ardenkjær-Larsen, J.Ø. Duus, M. Karlsson, M.H. Lerche, Detection of low-populated reaction intermediates with hyperpolarized NMR, Chem. Commun. (2009) 5168. doi:10.1039/b910626j.

[6] Y. Lee, G.S. Heo, H. Zeng, K.L. Wooley, C. Hilty, Detection of Living Anionic Species in Polymerization Reactions Using Hyperpolarized NMR, J. Am. Chem. Soc. 135 (2013) 46364639. doi:10.1021/ja4001008.

[7] R.R. Ernst, G. Bodenhausen, A. Wokaun, Principles of nuclear magnetic resonance in one and two dimensions, Clarendon Press; Oxford University Press, Oxford [Oxfordshire]: New York, 1987.

[8] L. Frydman, T. Scherf, A. Lupulescu, The acquisition of multidimensional NMR spectra within a 
single scan, Proc. Natl. Acad. Sci. 99 (2002) 15858-15862. doi:10.1073/pnas.252644399.

[9] L. Frydman, D. Blazina, Ultrafast two-dimensional nuclear magnetic resonance spectroscopy of hyperpolarized solutions, Nat. Phys. 3 (2007) 415-419. doi:10.1038/nphys597.

[10] P. Giraudeau, L. Frydman, Ultrafast 2D NMR: An Emerging Tool in Analytical Spectroscopy, Annu. Rev. Anal. Chem. 7 (2014) 129-161. doi:10.1146/annurev-anchem-071213-020208.

[11] E. Kupče, T. Nishida, R. Freeman, Hadamard NMR spectroscopy, Prog. Nucl. Magn. Reson. Spectrosc. 42 (2003) 95-122. doi:10.1016/S0079-6565(03)00022-0.

[12] H.-Y. Chen, C. Hilty, Hyperpolarized hadamard spectroscopy using flow NMR, Anal. Chem. 85 (2013) 7385-7390. doi:10.1021/ac401293n.

[13] W.A. Anderson, R. Freeman, Influence of a Second Radiofrequency Field on High-Resolution Nuclear Magnetic Resonance Spectra, J. Chem. Phys. 37 (1962) 85. doi:10.1063/1.1732980.

[14] A.L. Bloom, J.N. Shoolery, Effects of Perturbing Radiofrequency Fields on Nuclear Spin Coupling, Phys. Rev. 97 (1955) 1261-1265. doi:10.1103/PhysRev.97.1261.

[15] J.L. Neves, B. Heitmann, N. Khaneja, S.J. Glaser, Heteronuclear decoupling by optimal tracking, J. Magn. Reson. 201 (2009) 7-17. doi:10.1016/j.jmr.2009.07.024.

[16] F. Schilling, S.J. Glaser, Tailored real-time scaling of heteronuclear couplings, J. Magn. Reson. 223 (2012) 207-218. doi:10.1016/j.jmr.2012.07.024.

[17] F. Schilling, L.R. Warner, N.I. Gershenzon, T.E. Skinner, M. Sattler, S.J. Glaser, Next-Generation Heteronuclear Decoupling for High-Field Biomolecular NMR Spectroscopy, Angew. Chem. Int. Ed. 53 (2014) 4475-4479. doi:10.1002/anie.201400178.

[18] N. Khaneja, T. Reiss, C. Kehlet, T. Schulte-Herbrüggen, S.J. Glaser, Optimal control of coupled spin dynamics: design of NMR pulse sequences by gradient ascent algorithms, J. Magn. Reson. 172 (2005) 296-305. doi:10.1016/j.jmr.2004.11.004.

[19] S.J. Glaser, U. Boscain, T. Calarco, C.P. Koch, W. Köckenberger, R. Kosloff, I. Kuprov, B. Luy, S. Schirmer, T. Schulte-Herbrüggen, D. Sugny, F.K. Wilhelm, Training Schrödinger's cat: quantum optimal control: Strategic report on current status, visions and goals for research in Europe, Eur. Phys. J. D. 69 (2015). doi:10.1140/epjd/e2015-60464-1.

[20] G. Zhang, F. Schilling, S.J. Glaser, C. Hilty, Chemical Shift Correlations from Hyperpolarized NMR Using a Single SHOT, Anal. Chem. 85 (2013) 2875-2881. doi:10.1021/ac303313s.

[21] S. Bowen, C. Hilty, Rapid sample injection for hyperpolarized NMR spectroscopy, Phys. Chem. Chem. Phys. 12 (2010) 5766. doi:10.1039/c002316g.

[22] M. Szwarc, “Living” Polymers, Nature. 178 (1956) 1168-1169. doi:10.1038/1781168a0.

[23] M. Szwarc, M. Levy, R. Milkovich, Polymerization Initiated by Electron Transfer to Monomer. A New Method of Formation of Block Polymers, J. Am. Chem. Soc. 78 (1956) 2656-2657. doi:10.1021/ja01592a101.

[24] K. Matsuzaki, Y. Shinohara, T. Kanai, Nuclear magnetic resonance studies on polymer carbanions, 1. Living polystyrene and its model compounds, Makromol. Chem. 181 (1980) 1923-1934. doi:10.1002/macp.1980.021810913.

[25] R.R. Ernst, Nuclear Magnetic Double Resonance with an Incoherent Radio-Frequency Field, J. Chem. Phys. 45 (1966) 3845. doi:10.1063/1.1727409. 